\title{
Cardiac Protection by Basic Fibroblast Growth Factor from Ischemia/Reperfusion-Induced Injury in Diabetic Rats
}

\author{
Jian XIAO, ${ }^{a, b, \#}$ Yanxia Lv, ${ }^{a, b, \#}$ Shaoqiang LiN,${ }^{a, b}$ Litai JIN,${ }^{a, b}$ Yi Zhang,${ }^{a, b}$ Xiaojie WANG, ${ }^{c}$ Jisheng MA, ${ }^{c}$ \\ Keqiong Hu, ${ }^{a, b}$ Wenke FEnG, ${ }^{a, d}$ Lu CAI, ${ }^{a, d}$ Xiaokun LI, ${ }^{a, b, c}$ and Yi TAN ${ }^{*, a, b, d}$ \\ ${ }^{a}$ Chinese-American Research Institute for Diabetic Complications, Wenzhou Medical College; ${ }^{b}$ Key Laboratory of \\ Biotechnology and Pharmaceutical Engineering, Wenzhou Medical College; Wenzhou 325035 China: ${ }^{c}$ Biopharmaceutics \\ and Bioreactor Research Center, Ministry of Education, Jilin Agriculture University; Changchun 130025, China: and \\ ${ }^{d}$ Department of Pediatrics, University of Louisville; Louisville, Kentucky 40202, U.S.A. \\ Received September 17, 2009; accepted December 1, 2009; published online December 7, 2009
}

Diabetes impairs the expression and function of endogenous growth factors, leading to increased cardiovascular events in diabetic patients. Supplementation of fibroblast growth factors (FGFs) protected the heart from ischemia/reperfusion (I/R)-induced injury in animal models. However, it has not yet been tested in diabetic heart. The present study was thus to clarify whether basic fibroblast growth factor (bFGF) could protect the heart from I/R-induced damage under diabetic conditions using a rat model. Male Sprague Dawley rats were used to induce diabetes by intraperitoneal injection of streptozotocin. Eight weeks later, I/R injury was generated in diabetic rats and age-matched non-diabetic rats. All I/R rats were administrated bFGF or saline through intramyocardial injection. Seven days after $\mathbf{I} / \mathbf{R}$, cardiac infarction, structural changes, cell death and blood vessel density, serum malondialdehyde (MDA) and cardiac enzyme lactate dehydrogenase (LDH) were examined. We found that I/R induced significant increases in the cardiac infarction, blood MDA contents and LDH activities, and the expression of caspase-3. Treatment of $I / R$ rats with $b F G F$ simultaneously with reperfusion significantly attenuated $I / R-$ induced pathological changes, along with a significant increase in the cardiac blood vessel density in both diabetic and non-diabetic rates. The protective effects of bFGF on I/R-induced cardiac injury in diabetic group are less than those in non-diabetic group. The results indicated that bFGF provide a protection of the heart against I/R-induced oxidative damage, cell death and infarction under diabetic conditions.

Key words basic fibroblast growth factor; diabetes; cardiac infarction; ischemia/reperfusion injury

Fibroblast growth factor (FGF) is a superfamily of at least 21 structurally and functionally relevant members characterized by a high affinity to heparin. ${ }^{1,2}$ Basic FGF (bFGF, also call FGF-2) was first extracted from bovine brain and characterized as a potent factor to stimulate NIH3T3 proliferation and mitogenesis in $1974 .{ }^{3)}$ Accumulating evidence demonstrates that FGFs, including bFGF, are multifunctional proteins exerting tissue mitogenic and protective actions against a variety of oxidative stresses. ${ }^{4-12)}$

Diabetes was found to impair endogenous defense mechanisms in the tissues. ${ }^{13-16)}$ For instance, diabetes inhibited the expression, and also induced glycosylation of several growth factors, including endogenous FGF. ${ }^{17-21)}$ Decreased serum levels of bFGF with other growth factors were found to increase cardiovascular events (including infarction, revascularization, stroke and peripheral vascular disease) in diabetic patients. $^{22)}$ Supplementation of exogenous FGFs attenuated diabetes-caused neuronal and gastric tissue damage. ${ }^{23,24)} \mathrm{We}$ have performed experimental and clinical studies, demonstrating that topical application of recombinant human acidic FGF (rhaFGF) and basic FGF (rhbFGF) promoted the healing of skin ulcer in diabetic patients, ${ }^{25)}$ and diabetic rats. ${ }^{26)}$

Cardiac infarction and dysfunction are life-threatening diseases. Subjects with diabetes experience an increased risk of cardiac infarction and cardiac failure compared to non-diabetic age-matched individuals. ${ }^{13,14,27,28)} \mathrm{We}$ and other groups have confirmed that supplementation or over-expression of FGFs can protect the heart from ischemia/reperfusion (I/R)induced injury in animal models and in patients. ${ }^{29-36)}$ However, whether FGFs can protect diabetic heart from I/Rinduced injury has not been addressed.
The present study was thus to clarify whether bFGF can protect the heart from I/R-induced damage under diabetic condition using a rat model. We found that administration of rhbFGF to diabetic rats indeed provided a protection against I/R-induced cardiac damage although the protective effect is slightly less in diabetic rats than that in non-diabetic rats.

\section{MATERIALS AND METHODS}

Animals Male Sprague Dawley rats purchased from Experimental Animal Center in Wenzhou Medical College (Wenzhou, Zhejiang, China), body weight $250 \pm 30 \mathrm{~g}$, and maintained under specific pathogen-free conditions at Wenzhou Medical College Animal Facility (Wenzhou, Zhejiang, China). All of the following animal procedures were approved by the College Animal Case and Use Committee, which is certified by the Chinese Association of Accreditation of Laboratory Animal Care.

Diabetic Model Type 1 diabetic models were induced with streptozotocin (STZ) as described in our previous report. ${ }^{26)}$ Briefly, male rats were given a single intraperitoneal (i.p.) injection of $55 \mathrm{mg} / \mathrm{kg} \mathrm{STZ}$ dissolved in sodium citrate buffer ( $\mathrm{pH} 4.5$ ), and $3 \mathrm{~d}$ later, whole-blood glucose obtained from rat tail-vein was monitored using SureStep Complete Blood Glucose monitor (LifeScan Inc., Milpitas, CA, U.S.A.). STZ-treated rats with whole-blood glucose levels higher than $11.1 \mathrm{~mm}(200 \mathrm{mg} / \mathrm{dl})$ were considered diabetic. Rats serving as vehicle controls (non-diabetic rats) were given the same volume of sodium citrate, as indicated in Fig. 1.

Ischemia/Reperfusion Injury Model and Drug Delivery 


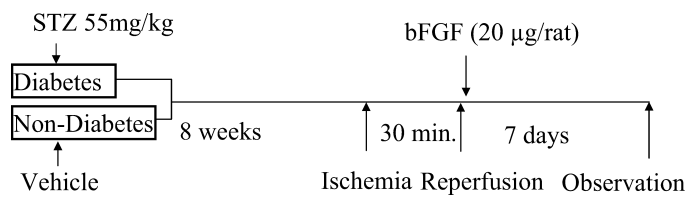

Fig. 1. The Outline of Overall Experimental Design

Diabetic and age-matched non-diabetic rats were used to generate cardiac I/R model at eight weeks after the onset of diabetes, as showed in Fig. 1. Under sufficient anesthesia with an i.p. injection of $5 \%$ chloral hydrate $(7 \mathrm{ml} / \mathrm{kg}$ body weight), rats were placed in a supine position and an endotracheal polyethylene (PE) 90 tubing was used to provide ventilation via a rodent ventilator (Harvard, South Natick, MA, U.S.A.) at a rate of 90 cycles per min. Oxygen (100\%) was provided to the in-flow of the ventilator, and the tidal volume was $3-4 \mathrm{ml} / 100 \mathrm{~g}$. The chest was opened by a lateral cut along the up-margin of the fourth rib. The left auricle was slightly retraced to expose the entire left anterior descending coronary artery (LADCA) system. Ligation was performed using a 6-0 silk suture passing through the space between the inferior border $1-3 \mathrm{~mm}$ of auticle of left atrium and pulmonary conus, and making a knot to occlude the coronary artery without veins occlusion. Coronary artery occlusion lasted $30 \mathrm{~min}$ and reperfusion was restarted by cutting the knot. Meanwhile, as outlined in Fig. 1, $0.1 \mathrm{ml}$ of rhbFGF ( $20 \mu \mathrm{g} / \mathrm{rat}$ ) or saline was administered by intramyocardial injection around the ligation site at four points $(25 \mu \mathrm{l} /$ point $)$, according to previous reports. ${ }^{34,35)}$ The chest wall was closed and the animal was removed from the respirator and kept warm by a heat lamp and allowed to breathe $100 \%$ oxygen via a nasal cone. The ventilator was removed after the selfbreath recovery and amikacin sulfate $(200000 \mathrm{U} / \mathrm{d} / \mathrm{rat})$ was given by intramuscular injection. The electrocardiography of the rats was monitored through all the surgical procedure. Therefore, there were four experimental groups with 6 rats per group without significant body weight variation: salinetreated non-diabetic rats with $\mathrm{I} / \mathrm{R}(\mathrm{Cn})$, saline-treated diabetic rats with $\mathrm{I} / \mathrm{R}(\mathrm{Dn})$, rhbFGF-treated non-diabetic rats with $\mathrm{I} / \mathrm{R}$ $(\mathrm{Cb})$, and rhbFGF-treated diabetic rats with $\mathrm{I} / \mathrm{R}(\mathrm{Db})$.

Assessment of Infarction Size Seven days after I/R, the cardiac left ventricles were harvested and sectioned transversely into five sections with one section being made at the site of the ligature, and the sections were weighed. The images of the representative sections were taken by a microscopy system (Nikon TE2000-S). Sections of the ventricle were then incubated in $1.5 \%$ triphenyltetrazolium chloride (TTC) for $15 \mathrm{~min}$ at $37^{\circ} \mathrm{C}$. After the above procedures, the heart was stained by brick red for normal tissue and pale white for infarct tissue. The size of infarction was determined by the following equations: infarction size $=$ weight of infarction/weight of total left ventricle $\times 100 \%$.

Detection of Lipid Peroxidation and Cardiac Damage Seven days after I/R, the carotid artery blood was collected to detect serum lipid peroxides by measuring malondialdehyde (MDA) concentrations, and cardiac enzyme lactate dehydrogenase (LDH) activities as an index of cardiac damage, using corresponding commercially-available assay kits (Nanjing Jiancheng Biological Institute, Nanjing, China). The concentration of MDA was expressed as nanomolar MDA
Table 1. Summary of the Protective Effect of bFGF on I/R-Induced Various Damages

\begin{tabular}{lccc}
\hline \hline \multirow{4}{*}{ Parameters } & \multicolumn{2}{c}{ Protective effects, \% } & \\
\cline { 2 - 3 } & $\begin{array}{c}\text { Non-diabetes }(n=6) \\
{[(\mathrm{Cb}-\mathrm{Cn})} \\
/ \mathrm{Cn} \times 100]\end{array}$ & $\begin{array}{c}\text { Diabetes }(n=6) \\
{[(\mathrm{Db}-\mathrm{Dn})} \\
/ \mathrm{Dn} \times 100]\end{array}$ & $\begin{array}{c}p \text { values } \\
\left(\chi^{2} \text { test }\right)\end{array}$ \\
\hline Cardiac infarction & 22.12 & 8.88 & $p=0.46$ \\
MDA concentration & 36.25 & 30.60 & $p=0.81$ \\
LDH activity & 46.5 & 36.7 & $p=0.69$ \\
Caspase-3 expression & 63.58 & 53.56 & $p=0.68$ \\
Cardiac blood vessel & 22.58 & 18.87 & $p=0.33$ \\
\hline
\end{tabular}

per milliliter serum and the LDH activity was expressed as LDH activity units per milliliter serum.

Histological Analysis and Immunohistochemistry Paraffin sections $(3 \mu \mathrm{m})$ of left ventricles were stained with hematoxylin and eosin (H\&E), and the morphological changes were examined under light microscope. Cardiac cell death was examined by immunohistochemical staining for activated form of caspase-3. Blood vessel density in the heart was identified by immunohistochemical staining for VIII factor.

For immunohistochemical staining, left ventricle sections were incubated with pepsin for $15 \mathrm{~min}$ at $37^{\circ} \mathrm{C}$, treated with $3 \% \mathrm{H}_{2} \mathrm{O}_{2}$ for $10 \mathrm{~min}$, and then incubated with polyclonal rabbit anti-active caspase-3 antibody $(1: 50)$ or polyclonal rabbit anti-VIII factor antibody $(1: 100)$ overnight at $4{ }^{\circ} \mathrm{C}$, respectively. Sections were then incubated for $30 \mathrm{~min}$ with horseradish peroxidase (HRP)-conjugated goat anti-rabbit immunoglobulin $\mathrm{G}(\mathrm{IgG})$ antibody. The antibody binding sites were visualized by incubation with DAB- $\mathrm{H}_{2} \mathrm{O}_{2}$ solution using a DAB kit (Maxvision, Fujian, China). Finally, sections were counterstained with hematoxylin.

Semi-quantitative analysis was performed with ten highpower fields $\left(0.08 \mathrm{~mm}^{2}\right)$ per slide to count for caspase-3 positive cells and capillary density in a double blind fashion. Six slides were analyzed per treatment group. The expression of caspase-3 was expressed as a percentage of caspase- 3 positive cells, which was calculated by positive cells/total cardiomyocytes counted in each high power field $\times 100$. The capillary density was expressed as the number of capillaries per high-power fields $\left(0.08 \mathrm{~mm}^{2}\right)(200 \times)$.

Statistical Analysis The data were analyzed using SPSS11.5 software and presented as mean \pm S.D. values from at least six rats in each group. The data were initially analyzed by one-way ANOVA analysis of variance and then in multiple comparisons with the Student $t$-test. Otherwise it is indicated for the specific assay (Table 1). Statistical significance was considered as $p<0.05$.

\section{RESULTS}

bFGF Prevented I/R-Induced Cardiac Infarction Size Seven days after $I / R$, the cardiac left ventricles were sectioned transversely and stained by TTC. The representative images of cardiac infarction from each group were shown in Fig. 2A, in which the intact cardiac tissue was stained brick red and the infarct tissue was stained pale white. Quantitative analysis of the infarction sizes (Fig. 2B) showed that diabetes 
A

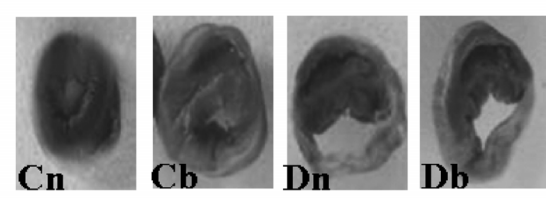

B

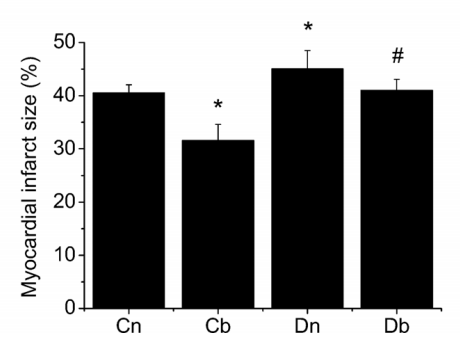

Fig. 2. Effects of bFGF on I/R-Induced Cardiac Infarction

The area of infarction in transverse sections of hearts was stained by TTC. All areas that were stained brick red were not at risk. Areas that appeared pale white were infarcted (A). The results of infarct size were calculated using ratio of weight of infarction to weight of total left ventricle (B). $* p<0.05$, vs. Cn; $\# p<0.05$, vs. Dn, $n=6$.

significantly increased I/R-induced cardiac infarction $(p<$ 0.05, Dn $v s . \mathrm{Cn}$ ), and rhbFGF protected heart from I/R injury under non-diabetic $(p<0.05, \mathrm{Cb} v s . \mathrm{Cn})$ and diabetic $(p<$ 0.05 , Dn $v s$. Db) conditions. However, the protective effect of rhbFGF on I/R-induced cardiac infarction in diabetic group is slightly less than that in non-diabetic hearts (Table 1) although there was no statistical difference $(p>0.05)$. This indicates that diabetes potentially impairs the protective action of rhbFGF against I/R-induced cardiac damage.

Histopathological examination of infarct tissues obtained from $I / R$ heart supported the finding of infarction sizes, showing that $\mathrm{I} / \mathrm{R}$ induced obvious cardiac damage and massive inflammatory cell infiltration (Fig. 3A), and diabetes further exaggerated these pathological changes (Fig. 3C). In contrast, most of cardiac muscles are undamaged with relative rare inflammatory cell infiltration in rhbFGF-treated non-diabetic (Fig. 3B) and diabetic rats (Fig. 3D), suggesting that rhbFGF protected heart from $\mathrm{I} / \mathrm{R}$ injury in diabetic and non-diabetic conditions.

bFGF Protected the Heart from I/R-Induced Oxidative Damage and Cell Death MDA is a product of lipid peroxidation induced by a diversity of oxidative injury. It has been used as a biomarker of cardiac oxidative damage. ${ }^{37)}$ The result presented in Fig. 4A shows the effect of rhbFGF on MDA concentrations in carotid artery blood. I/R dramatically elevated the MDA concentrations in non-diabetic rats $7 \mathrm{~d}$ after reperfusion. Diabetes further enhanced the I/R-induced MDA accumulation. However, the concentration of MDA in the rhbFGF-treated myocardium was significantly lower than those without rhbFGF-treatment in both diabetic and non-diabetic conditions (Fig. 4A). The protective effect of rhbFGF on $\mathrm{I} / \mathrm{R}$-induced MDA in diabetic group (30.6\%) is slightly lower than that in non-diabetic group (36.25\%), although there was no statistical difference (Table $1, p>0.05$ ).

The release of the cardiac enzyme LDH as an index of cardiac cell damage was measured in the carotid artery blood collected from the different groups. I/R caused a significant increase in $\mathrm{LDH}$, and diabetes further enhanced this damage (Fig. 4B). Administration of rhbFGF immediately after start-
A
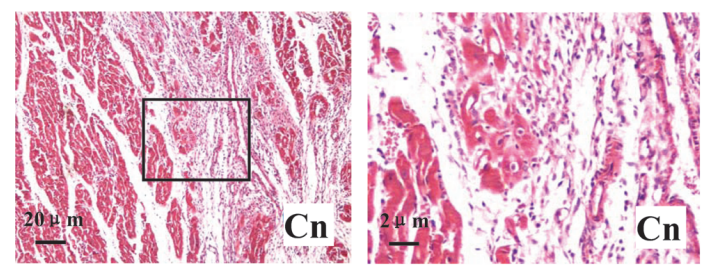

B
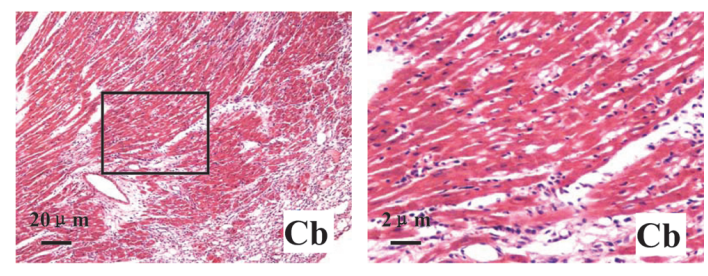

C

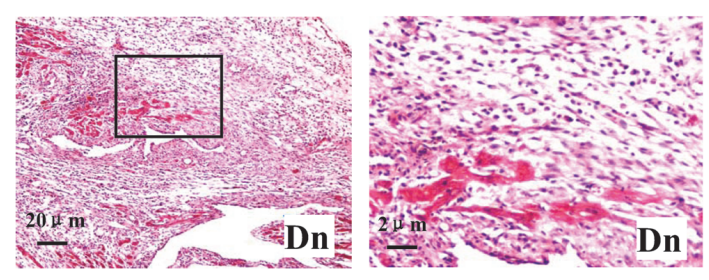

D

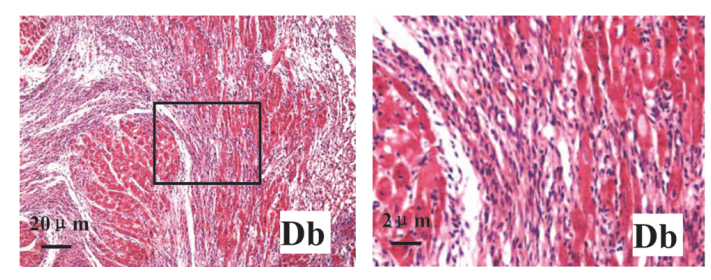

Fig. 3. bFGF Protected Heart from I/R-Induced Structural Changes

$H \& E$ staining indicates that $I / R$ induced obvious cardiomyocyte necrosis, cardiac fiber dissolution, and massive inflammatory cell infiltration (A), and diabetes worsened these changes (C). Administration of rhbFGF protected the hearts from I/R injury, indicated by less inflammatory cell infiltration in the risk areas (B). Diabetes slightly attenuated the protective effect of rhbFGF (D) as compared to that in non-diabetic rats (B). High-power views of sub-areas of left panels were showed in right panels.

ing reperfusion significantly prevented I/R-induced increase of the LDH level in carotid artery blood for both diabetic and non-diabetic rats (Fig. 4B). However, similar to the protective effect of rhbFGF on MDA levels, the protective effect of rhbFGF on I/R-induced increase in LDH in the blood in diabetic group (36.7\%) is also slightly, even though not statistically, lower than that in non-diabetic group $(46.50 \%$, Table 1 , $p>0.05$ ).

To define the protective effect of rhbFGF against I/Rinduced cardiac cell death, immunohistochemical staining of the cleaved caspase- 3 as an index of cell death was performed. Quantitative analysis showed that caspase-3 positive cells are significantly increased in infarct tissues (Fig. 4C, Cn and Dn). A protective effect by rhbFGF is observed, although this effect in diabetic group (53.56\%) is slightly, but not statistically, lower than that in non-diabetic group $(63.58 \%$, Table $1, p>0.05$ ).

bFGF Improved Cardiac Blood Vessels Formation The blood vessel density in $\mathrm{I} / \mathrm{R}$ injured heart was assessed by counting the factor VIII positive staining vessels (Fig. 5A). Results showed that the density of blood vessels in rat treated with rhbFGF was significant higher than that of vehicletreated group on day 7 after I/R $(p<0.05)$. Diabetes significantly inhibited the blood vessel formation in the heart after $\mathrm{I} / \mathrm{R} \quad(p<0.05)$ (Fig. 5B). Treatment of diabetic rats with 
A

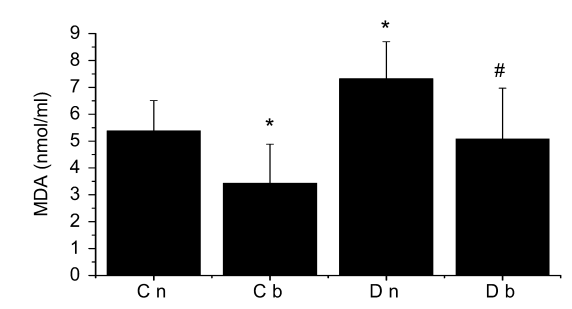

B

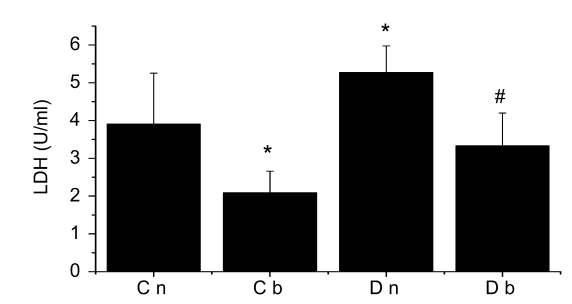

C

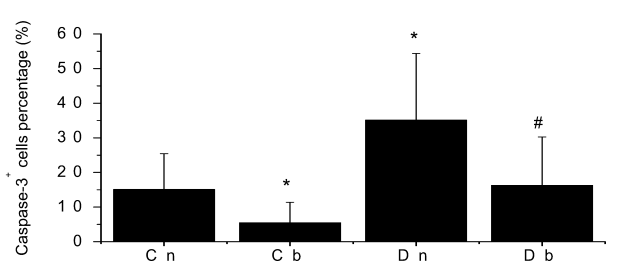

Fig. 4. Effects of bFGF on I/R-Induced Increase in MDA Concentration, LDH Activity and Caspase-3 Expression in the Heart

The carotid artery blood was collected to detect malondialdehyde (MDA) concentrations (A) and lactate dehydrogenase (LDH) activities (B) at $7 \mathrm{~d}$ after I/R. Left ventricle sections were stained by caspase 3 for cardiac cell death detection, which was expressed as the ratio of caspase- 3 positive cells to total cardiomyocytes, as described in Materials and Methods. $* p<0.05$, vs. Cn; $\# p<0.05$, vs. Dn, $n=6$.

A
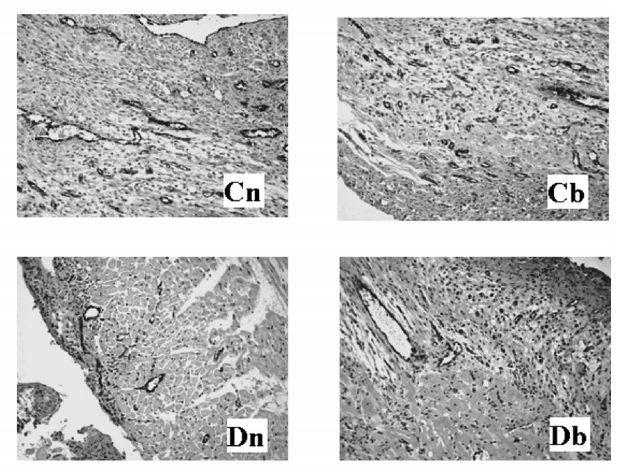

B

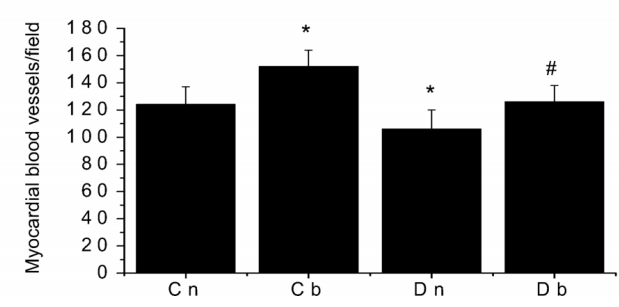

Fig. 5. bFGF Improved Blood Vessel Formation in I/R Heart

Left ventricle sections were stained with factor VIII for identifying blood vessels (A). Vessels were counted under high-power field $(200 \times)$ in a randomized fashion with 6 slides per animal from six rats in each group. $* p<0.05$, vs. Cn; $\# p<0.05, v s$. Dn.

rhbFGF immediately after $\mathrm{I} / \mathrm{R}$ significantly enhanced the blood vessel formation showing $18.87 \%$ and $22.58 \%$ enhancing effects in diabetic group and non-diabetic group, respectively (Table $1, p>0.05$ ).

\section{DISCUSSION}

In the present study, the cardioprotective effects of rhbFGF administered simultaneously with reperfusion after $30 \mathrm{~min}$ ischemia was investigated in diabetic rats. We confirmed that diabetes further enhanced $\mathrm{I} / \mathrm{R}$-induced cardiac infarction size, inflammatory response and cardiac damage. We provided the following novel findings: (1) Administration of bFGF immediately after reperfusion significantly protected the heart from I/R-induced injuries. (2) Treatment of I/R diabetic rats with rhbFGF significantly enhanced the blood vessel formation. (3) In generally speaking, cardiac protective effects of rhbFGF on $\mathrm{I} / \mathrm{R}$-induced pathogenesis in diabetic rats are slightly less than those in non-diabetic rats.

FGF plays both antioxidant and angiogenic functions. ${ }^{4-12)}$ Pre-treatment of animals with recombinant FGF without mitogenic action significantly protected the heart from I/R-induced damage in non-diabetic models. ${ }^{30,32)}$ The cultured cardiomyocytes transfected with non-mitogenic FGF gene and Langendorff heart perfused with non-mitogenic FGF were found to be significantly protected from oxidative damage such as hydrogen peroxide- and I/R-induced damage. ${ }^{29)}$ Under diabetic condition, superoxide dismutase (SOD) plus catalase was also found to significantly improve cardiac postischemic recovery. ${ }^{38)}$ These studies suggest that oxygen derived free radicals may mediate reperfusion-induced contractile dysfunction and ventricular fibrillation in diabetic hearts following brief episodes of cardiac ischemia. Consistent with these early studies, we demonstrated the protective effect of rhbFGF on I/R-induced increase of the blood MDA contents and cardiac cytotoxic effects (blood LDH levels and cardiac caspase-3 activation) in non-diabetic rats. Furthermore, we also demonstrated for the first time that rhbFGF significantly protected the heart from I/R-induced these oxidative damages under diabetic conditions (Fig. 4).

A short duration of hyperglycemia may protect the heart from I/R-induced injury, ${ }^{39)}$ but most studies have demonstrated that diabetes enhances cardiac damage, infarction and contractile dysfunction. ${ }^{13,14,27,28)}$ Under normal condition, pathophysiological stimuli that increase oxidative stress can up-regulate certain protective molecules such as heme oxygenase (HO)-1, a cytoprotective heme-degrading enzyme, and antioxidants including SOD, catalase and metallothionein in the heart. ${ }^{40)}$ It was found that HO-1 expression was increased in the mouse hearts subjected to I/R, but I/R-induced up-regulation of HO-1 expression in non-diabetic mice was absent in diabetic hearts. ${ }^{13)}$ Therefore, the absent expression of HO-1 made the heart more susceptible to I/R-induced damage and diabetes worsened these injuries. ${ }^{13,14)}$ These results suggest that diabetes could impair the endogenous and protective mechanisms in the heart, making the heart more sensitive to subsequent oxidative damage. . $^{13,14,27,28)}$

I/R-induced cardiac damages include three periods: (1) acute oxidative damages during ischemic period, (2) acute oxidative damage shortly after reperfusion, and (3) chronic damages after reperfusion. Cardiac infarction is related to both acute phase cell death and the post-I/R chronic cell death. The chronic cell death may be induced by the post-I/R oxidative damage and associated inflammatory response, but may be prevented by quick angiogenic recovery. ${ }^{41,42)} \mathrm{Al}$ though administration of bFGF before heart ischemia was 
found to be an effective approach to prevent the heart from $\mathrm{I} / \mathrm{R}$-induced damage, it may be not a practical approach since it will be difficult to predict the occurrence of heart stroke (ischemia). However, administration of the efficient cardioprotective reagent such as bFGF at the cardiac reperfusion for heart stroke patient would be a feasible approach. In the present study, therefore, bFGF was given simultaneously with cardiac reperfusion (Fig. 1). This is mainly to explore the preventive effect of bFGF on I/R-induced chronic damage. Therefore, antioxidant and angiogenic actions of rhbFGF should both play critical roles in determining the prevention of I/R-induced cardiac oxidative damage, inflammation, cell death and infarction. ${ }^{43}$ ) To support this notion, we demonstrated that rhbFGF treatment significantly prevented I/R-induced oxidative damage and cell death, and also significantly increased blood vessel densities in $\mathrm{I} / \mathrm{R}$ hearts of both non-diabetic and diabetic rats (Figs. 4, 5). Consequently, bFGF significantly prevented I/R-induced cardiac infarction (Fig. 2).

However, the protective effect of bFGF on I/R-induced cardiac injury, especially under diabetic conditions, is a complicated process and may include multiple mechanisms. Except we proposed anti-oxidative and angiogenic action in the above, other functions of bFGF such as cardiac stem cell mobilization and differentiation ${ }^{44)}$ might also play important role in I/R-induced cardiac injury protection. These need to be defined in the further studies.

In summary, we present the first evidence that administration of rhbFGF immediately after reperfusion can significantly prevent $\mathrm{I} / \mathrm{R}$-induced cardiac damage in diabetic rats. It is known that antioxidant and angiogenic action of FGF are mediated by signaling pathways that involves in several other kinases, such as Akt, extracellular signal-regulated kinase (Erk1/2), and vascular endothelial growth factor (VEGF). ${ }^{13-16,45)}$ As mentioned above, diabetes impairs several antioxidants and growth factors, ${ }^{17-21)}$ administration of single exogenous rhbFGF alone is unable to overcome all these defectives, and consequently offered a slightly less protection in diabetic rats as compared to that in non-diabetic rats. Therefore, combination of bFGF with other growth factors may be a potential approach to optimize the protective effect of bFGF against $\mathrm{I} / \mathrm{R}$-induced cardiac injury ${ }^{46)}$ even under diabetic conditions, which will be warranty in the future studies.

Acknowledgements This work was supported, in part, by research grants from Ministry of Education Incubation Foundation of Technology Innovative Project (No. 706018), the Program of New Century Excellent Talents in University and Zhejiang Provincial Program for the Cultivation of Highlevel Innovative Health Talents (to XL), Zhejiang Provincial Medicine and Health Foundation and NSFC (2006A099 and 30971209 , to YT); ADA (02-07-JFA-10; 05-07-CD-02, to LC), and JDRF (5-2006-382) and also by Start-Up Fund from Wenzhou Medical College for the Chinese-American Research Institute for Diabetic Complications (to LC \& XL).

\section{REFERENCES}

1) Fu X., Cuevas P., Gimenez-Gallego G., Sheng Z., Tian H., Chin. Med. $J$. (Engl.), 108, 209-214 (1995).

2) Detillieux K. A., Sheikh F., Kardami E., Cattini P. A., Cardiovasc. Res., 57, 8-19 (2003).
3) Gospodarowicz D., Nature (London), 249, 123-127 (1974).

4) Epstein S. E., Fuchs S., Zhou Y. F., Baffour R., Kornowski R., Cardiovasc. Res., 49, 532-542 (2001).

5) Klahr S., Morrissey J. J., Kidney Int. Suppl., 75, S7-S14 (2000).

6) Waltenberger J., Circulation, 96, 4083-4094 (1997).

7) Detillieux K. A., Cattini P. A., Kardami E., Can. J. Physiol. Pharmacol., 82, 1044-1052 (2004).

8) Finch P. W., Rubin J. S., Adv. Cancer Res., 91, 69-136 (2004).

9) Braun S., Auf Dem Keller U., Steiling H., Werner S., Philos. Trans. $R$. Soc. Lond. B Biol. Sci., 359, 753-757 (2004).

10) Danilenko D. M., Toxicol. Pathol., 27, 64-71 (1999).

11) Podolsky D. K., J. Gastroenterol., 32, 122-126 (1997).

12) Klagsbrun M., Semin. Cancer Biol., 3, 81-87 (1992).

13) Di Filippo C., Marfella R., Cuzzocrea S., Piegari E., Petronella P., Giugliano D., Rossi F., D'amico M., Diabetes, 54, 803-810 (2005).

14) Liu X., Wei J., Peng D. H., Layne M. D., Yet S. F., Diabetes, 54, 778 784 (2005)

15) Xia Z., Guo Z., Nagareddy P. R., Yuen V., Yeung E., Mcneill J. H., Eur. J. Pharmacol., 544, 118-125 (2006).

16) Song P., Wu Y., Xu J., Xie Z., Dong Y., Zhang M., Zou M. H., Circulation, 116, 1585-1595 (2007).

17) Hart A. W., Baeza N., Apelqvist A., Edlund H., Nature (London), 408, 864-868 (2000).

18) Facchiano F., D'arcangelo D., Russo K., Fogliano V., Mennella C., Ragone R., Zambruno G., Carbone V., Ribatti D., Peschle C., Capogrossi M. C., Facchiano A., Mol. Endocrinol., 20, 2806-2818 (2006).

19) Mclennan S. V., Kelly D. J., Schache M., Waltham M., Dy V., Langham R. G., Yue D. K., Gilbert R. E., Kidney Int., 72, 481-488 (2007).

20) Tanii M., Yonemitsu Y., Fujii T., Shikada Y., Kohno R., Onimaru M., Okano S., Inoue M., Hasegawa M., Onohara T., Maehara Y., Sueishi K., Circ. Res., 98, 55-62 (2006).

21) Facchiano F., Lentini A., Fogliano V., Mancarella S., Rossi C., Facchiano A., Capogrossi M. C., Am. J. Pathol., 161, 531-541 (2002).

22) Yeboah J., Sane D. C., Crouse J. R., Herrington D. M., Bowden D. W., Dis. Markers, 23, 173-178 (2007).

23) Takeuchi K., Takehara K., Tajima K., Kato S., Hirata T., J. Pharmacol. Exp. Ther., 281, 200-207 (1997).

24) Nakae M., Kamiya H., Naruse K., Horio N., Ito Y., Mizubayashi R., Hamada Y., Nakashima E., Akiyama N., Kobayashi Y., Watarai A., Kimura N., Horiguchi M., Tabata Y., Oiso Y., Nakamura J., Diabetes, 55, 1470-1477 (2006).

25) Tan Y., Xiao J., Huang Z., Xiao Y., Lin S., Jin L., Feng W., Cai L., Li X., J. Health Sci., 54, 423-440 (2008).

26) Wang W., Lin S., Xiao Y., Huang Y., Tan Y., Cai L., Li X., Life Sci., 82, 190-204 (2008).

27) Song Y., Song Z., Zhang L., Mcclain C. J., Kang Y. J., Cai L., Cardiovasc. Toxicol., 3, 363-372 (2003).

28) Xiao C. Y., Chen M., Zsengeller Z., Szabo C., J. Pharmacol. Exp. Ther., 310, 498-504 (2004).

29) Li X. K., Lin Z. F., Li Y., Hu S., Tan Y., Huang Z., Meng J., Liang L. M., Xiao J., Qu J., Cai L., Cardiovasc. Pathol., 16, 85-91 (2007).

30) Cuevas P., Carceller F., Lozano R. M., Crespo A., Zazo M., GimenezGallego G., Growth Factors, 15, 29-40 (1997).

31) Cuevas P., Carceller F., Martinez-Coso V., Asin-Cardiel E., GimenezGallego G., Eur. J. Med. Res., 5, 145-149 (2000).

32) Cuevas P., Reimers D., Carceller F., Martinez-Coso V., RedondoHorcajo M., Saenz De Tejada I., Gimenez-Gallego G., Eur. J. Med. Res., 2, 465-468 (1997).

33) Palmen M., Daemen M. J., De Windt L. J., Willems J., Dassen W. R., Heeneman S., Zimmermann R., Van Bilsen M., Doevendans P. A., J. Am. Coll. Cardiol., 44, 1113-1123 (2004).

34) Schumacher B., Pecher P., Von Specht B. U., Stegmann T., Circulation, 97, 645-650 (1998).

35) Nishida S., Nagamine H., Tanaka Y., Watanabe G., Circ. J., 67, 334 339 (2003).

36) Kawasuji M., Nagamine H., Ikeda M., Sakakibara N., Takemura H., Fujii S., Watanabe Y., Ann. Thorac. Surg., 69, 1155-1161 (2000).

37) Van Linthout S., Riad A., Dhayat N., Spillmann F., Du J., Dhayat S., Westermann D., Hilfiker-Kleiner D., Noutsias M., Laufs U., Schultheiss H. P., Tschope C., Diabetologia, 50, 1977-1986 (2007).

38) Pieper G. M., Cardiovasc. Res., 22, 916-926 (1988).

39) Ma G., Al-Shabrawey M., Johnson J. A., Datar R., Tawfik H. E., Guo D., Caldwell R. B., Caldwell R. W., Naunyn Schmiedebergs Arch. 
Pharmacol., 373, 415-427 (2006).

40) Yin X., Wu H., Chen Y., Kang Y. J., Biochem. Pharmacol., 56, 87—93 (1998).

41) Loesser K. E., Kukreja R. C., Kazziha S. Y., Jesse R. L., Hess M. L., Cardioscience, 2, 199-216 (1991).

42) Moens A. L., Claeys M. J., Timmermans J. P., Vrints C. J., Int. J. Cardiol., 100, 179-190 (2005).

43) Bougioukas I., Didilis V., Ypsilantis P., Giatromanolaki A., Sivridis E., Lialiaris T., Mikroulis D., Simopoulos C., Bougioukas G., Cardiovasc.
Pathol., 16, 63-68 (2007).

44) Takehara N., Tsutsumi Y., Tateishi K., Ogata T., Tanaka H., Ueyama T., Takahashi T., Takamatsu T., Fukushima M., Komeda M., Yamagishi M., Yaku H., Tabata Y., Matsubara H., Oh H., J. Am. Coll. Cardiol., 52, 1858-1865 (2008).

45) Gross E. R., Hsu A. K., Gross G. J., Diabetes, 56, 127-136 (2007).

46) Kano M. R., Morishita Y., Iwata C., Iwasaka S., Watabe T., Ouchi Y., Miyazono K., Miyazawa K., J. Cell Sci., 118, 3759-3768 (2005). 